beyond'. It is recognised that the prevalence of children with life-limiting conditions (LLC) is increasing. In Ireland the majority of children with LLC are cared for by paediatricians working in hospitals with no direct access to specialist paediatric palliative care(PPC) services.

This study was part of a learning needs assessment of paediatrician's training and experience in PPC.

Methods Consultants and Specialist Registrars in Paediatrics were approached by email and asked to complete a questionnaire regarding their learning needs in PPC. The Quality of Care Collaborative for Australia in PPC Project Research Study Group Learning Needs Assessment template was used with permission.

The questionnaire included both multiple choice and freetext questions. Quantitative data from the study has been reported elsewhere. Thematic analysis based on the approach of Braun and Clarke (2006) was used to analyse data provided in the free-text answers.

Results 114 paediatricians responded to the survey, 72 respondents (63\%) provided free-text answers to the question 'Reflecting on your most recent clinical interactions with children requiring palliative care support. What were the challenges?'

On analysis, three overarching themes emerged, 'Best interests of the child', 'Inadequate training and confidence' and 'Co-ordinating care'.

'Best interests of the child' referred to the challenges experienced in ensuring that best interests of the child remain at the core of all decisions made when providing palliative care. This overarching theme included two sub-themes 'Complex communication' which referred to the challenges in managing parental expectations in increasingly technologically advanced healthcare systems and 'First do no harm' referring to the frequent ethical dilemmas that arise in PPC.

'Inadequate adequate training and confidence' referred to lack of specific education in complex symptom management and also to lack of familiarity with community based healthcare services. The theme of 'coordinating care' encompassed the need to liaise with community services but also the need to provide holistic care to the whole family which may be unfamiliar paediatricians working in an acute hospital setting. Conclusion This study explores the challenges experienced by paediatricians providing palliative care to children with LLCs. The themes provide insight into those experiences and provides valuable direction to support the development of future educational programmes in this important and rapidly evolving paediatric sub-speciality.

\section{GP88 ENHANCING SERVICES FOR VULNERABLE CHILDREN AND FAMILIES THROUGH PUBLIC HEALTH NURSES PARTICIPATION IN A WORKFORCE CAPACITY BUILDING INITIATIVE AND INTERAGENCY AND PARTNERSHIP IN A LOW-INCOME IRISH COMMUNITY}

${ }^{1}$ Lynn Buckley*, ${ }^{2}$ Margaret Curtin, ${ }^{1}$ Catherine Maguire, 'Grace Walsh, '2Louise Gibson, ${ }^{1}$ Katherine Harford. 'Young Knocknaheeny, Cork, Ireland; ' University College Cork, Cork, Ireland

\subsection{6/archdischild-2019-epa.153}

Background The provision of high-quality support and care from pregnancy to age three can influence lifelong health and social outcomes. This is particularly the case for children born into intergenerational poverty. Evidence suggests that progressive, targeted-universal approaches are most effective in enhancing early development outcomes, particularly when delivered through community-based infant health and wellbeing services.

In Ireland, Public Health Nurses (PHNs) provide the core nursing and midwifery care in the community working from a 'cradle to grave' model that span the lifecycle. This model requires PHNs to provide specialist early years support to vulnerable children and families within a largely generalist role. Additional training and engagement in innovative partnership can support PHNs to fulfil this role. Young Knocknaheeny $(\mathrm{YK})$ is an interagency collaboration aiming to reduce child poverty and enhance child development (age 0 to 6) across four low-income Irish neighbourhoods. The programme is underpinned by an innovative Infant Mental Health (IMH) framework. Locally-based PHNs are key partners in YK and have engaged in a variety of initiatives. These included: an intensive IMH training and coaching programme; inter-disciplinary prevention and early intervention using an IMH framework, through a local KidScope paediatric clinic; working with YK Oral Language Officers to offer accessible speech, language and communication support to all parents with babies attending the 7 to 9 month developmental assessments (Babbling Babies clinic).

Aims The overall aim was to explore the impact of training, capacity-building and partnership working on services delivered by PHNs to vulnerable children and families.

Methodology This study formed part of a process evaluation of the YK programme undertaken between 2015 and 2017. A mixed methods approach was adopted. YK and KidScope administrative databases were analysed. Qualitative methods were used to gain PHNs perspectives on IMH training and their engagement with YK, Babbling Babies and KidScope.

Results Twenty-four PHNs participated in IMH training and on-going mentoring. $63 \%$ of children born in the area were seen at the Babbling Babies clinic. PHNs reported the IMHinformed 'common language' enhanced the quality of their interactions with community practitioners and with families. The partnership process further facilitated PHNs engagement with 'hard-to-reach' families.

Discussion/Conclusion The YK programme enhanced the capacity of PHNs to provide high-quality support to vulnerable families in the critical early years. The evaluation suggests that a shift in the current 'cradle to grave' PHN model towards a specialist early years PHN could improve health and well-being outcomes for children/families.

\section{GP89 'TICK WITH TREAT'}

${ }^{1}$ Enrica Manca* ${ }^{*}$ Agostino Petraccaro, ${ }^{2}$ Carmela De Meco, ${ }^{2}$ Antonio Marseglia, ${ }^{1}$ Rossella Giorgio, ${ }^{1}$ Rosa Canestrale, ${ }^{1}$ Sofia Siena, ${ }^{2}$ Michele Sacco, ${ }^{2}$ Massimo PettoelloMantovani. 'Residency program in Pediatrics, University of Foggia, Foggia, Italy; ${ }^{2}$ Department of Pediatrics, Pediatric Unit, 'Casa Sollievo della Sofferenza' Scientific Institute, University of Foggia, San Giovanni Rotondo, Foggia, Italy

\subsection{6/archdischild-2019-epa.154}

Ticks can determine various local reactions, among which scarring and nonscarring inflammatory alopecia. We describe a case of nonscarring alopecia in a two-year-old girl of Romanian origin who reported a recent history of tick bite. She referred to our pediatric department with diffuse alopecia of the scalp, in which there was an erythematous 